\title{
Schätzung des Prüfniveaus metabolischer Merkmale beim Glukose- Toleranz-Test (GTT) an Jungbullen
}

Herrn Prof. Dr. agr. habil. Dr. h. c. mult. Georg Schönmuth zum 75. Geburtstag gewidmet

\begin{abstract}
Summary
Title of the paper: Level estimation of metabolic parameters in the glucose tolerance test (GTT) of young bulls

The metabolic parameters of the glucose tolerance test are suitable for an additional recommendation for the valuation of the breeding bulls before the start of the offspring's test could be given. Very important is the level of glucose half live to the begin of lactation of the cows. The other limit is the hyperglycemias greater than 10 $\mathrm{mmol} / \mathrm{l}$ ( or $180 \mathrm{mg} / \mathrm{dl}$ ) in the glucose level in the blood. The glucose half live measured nearly 48 minutes is comparable in the $8^{\text {th }}$ week post partum in lactation of cows with the young bulls in the age one year. Their combination with the pedigree breeding value is leading to increase of information, that could be utilised to the selection of improper bulls if the present results were confirmed.
\end{abstract}

Key Words: cattle, glucose, young bulls, cows

\section{Zusammenfassung}

Die metabolischen Parameter des Glukosetoleranztestes (GTT) sind für eine zusätzliche Empfehlung zur Bewertung von Zuchtbullen vor der Nachkommenprüfung geeignet. Wichtig ist das Niveau der Glukosehalbwertzeit zu Beginn der Laktation der Kuh. Ein anderes Limit ist die renale Resorptionsschwelle über $10 \mathrm{mmol} / \mathrm{l}$ ( oder $180 \mathrm{mg}$ / dl ) im Glukoseniveau im Blut. Die gemessene Glukose-Halbwertzeit um 48 Minuten in der 8. Laktationswoche der Kuh ist vergleichbar mit der der Jungbullen im Alter von einem Jahr. Ihre Kombination mit dem Pedigreezuchtwert führt zu einem Informationszuwachs, der bei der Auswahl der Testbullen genutzt werden kann.

Schlüsselwörter: Rind, Glukose, Jungbullen, Kühe, Milch

\section{Einleitung}

Die Züchter sind an frühzeitigen Informationen zur Bewertung wachsender Jungbullen insbesondere beim langen Generationsintervall des Rindes interessiert. Die gemeinsame Betrachtung genetischer und physiologischer Aspekte erscheint zur Aufklärung der Zusammenhänge geboten. Die Glukoseproduktion der Tiere ist eine wesentliche Voraussetzung für die Milchproduktion und Gesundheit. Eine hohe Milchleistung bei gleichzeitiger stabiler Stoffwechselgesundheit mit ungestörter Fruchtbarkeit der Milchkuh wird in entscheidendem Maße von einer ausgewogenen Energieverteilung im Körper beeinflusst. Für den ungestörten Ablauf der Körperfunktionen wird der Glukosespiegel im Blut der Wiederkäuer mit 35 - 55 mg Glukose/100 ml annähernd 
konstant gehalten (KIRCHGESSNER, 1997). Enzyme, Hormone, Kontrollproteine und Transporter tragen zur enormen Syntheseleistung von 3-4 kg Glukose bei. Insulin nimmt eine zentrale Stellung bei der Regulation des Energiestoffwechsels der Rinder ein. Es besteht ein enger Zusammenhang zwischen dem Insulin als regelnde und der Glukose als geregelte Größe. Im Stoffwechsel der Kuh wird Glukose für verschiedene Leistungen benötigt. Sie ist erforderlich für die Synthese der Lactose, für die ATP-Bildung über die Glycolyse und den Tricarbonsäurezyklus, für die Bereitstellung von NADPH und Ribose über den Pentosephosphatweg und für die Bildung von Glycerol für die Fettsynthese. Umfangreiche Untersuchungen von STAUFENBIEL (1993) zeigten insbesondere die Balance zwischen Katabolismus und Anabolismus im Fettstoffwechsel.

Die Messung der Glukose- und Insulinreaktion während einer induzierten Stoffwechselbelastung bietet eine Möglichkeit der Quantifizierung des Stoffwechselreaktionsvermögens von Rindern (BURKERT, 1998). Eine Belastungssituation entsteht während einer durch Nahrungsentzug eingeleiteten Hungerperiode (SEJRSEN et al., 1984; OLBRICH-BLUDAU et al., 1993; MIN et al., 1993; ROBINSON et al., 1994; MÜLLER et al., 1997) oder durch die intravenöse Injektion von Hormonen oder Metaboliten wie GRF, Adrenalin, Arginin, Glukagon und Glukose (EULITZ MEDER et al., 1988; MACKENZIE et al., 1988; REINICKE, 1993; XING et al., 1993; LØVENDAHL et al., 1995; BURKERT, 1998; SØRENSEN et al., 2000; PANICKE et al., 2001). Hierzu liegen keine durchgängigen Untersuchungen mit einem breiten Merkmalsspektrum an Rindern unterschiedlicher Altersklassen oder gleicher bzw. vergleichbarer Tiere über einen längeren Zeitraum von wenigstens fünf Jahren vor. Bei zusammenfassender Betrachtung sind die Glukosekonzentration im Blut sowie der Wachstumshormonspiegel als potentielle Indikatoren anderen physiologischen Merkmalen wie Freie Fettsäuren, Harnstoff, Thyroxin, Prolactin, Somatropin oder IGF-1 bezüglich der Beziehungen der metabolischen Merkmale zu den Leistungen der Kühe und Bullen überlegen (SEELAND, SCHÖNMUTH et al., 1984; REINICKE und SEELAND, 1985; OLDENBROEK et al., 1997, PANICKE et al., 2001). In Übereinstimmung der eigenen Ergebnisse (PANICKE et al., 2000 a, b, 2001, 2002) mit denen von SOERENSEN und LØVENDAHL (2000), REINICKE et al. (1993), SEJRSEN et al. (1984) verdient die Regulation der Glukosekonzentration weiter untersucht zu werden Die Insulinfunktion und Glukosekonzentration im Blut kann mit Hilfe des Glukosetoleranztestes (GTT) erfasst werden. Der Erfolg wird vom vergleichbaren altersphysiologischen und metabolischen Status bestimmt. Das Ziel der Untersuchung ist es, ein vergleichbares Prüfniveau für den GTT bei wachsenden Jungrindern abzuleiten.

\section{Material und Methode}

Für den Vergleich der Parameter des Glukose-Toleranz-Testes (GTT) von Jungbullen mit Kühen im Laktationsverlauf stand ein Material von 292 Jungbullen im dritten Lebenshalbjahr aus drei Geburtsjahrgängen und 120 Kühen im Stadium von 8 Wochen ante partum bis 36 Wochen post partum zur Verfügung. Der intravenöse Glukosetoleranztest (GTT) wurde von WEBER (2003) in 2 Bullen-Aufzuchtstationen an Bullen der Rasse Deutsche Holsteins und von REINICKE (1993) in einer Kuhherde durchgeführt. Die Bullen und Kühe konnten seit der letzten Fütterung am Nachmittag des Vortages lediglich Wasser aufnehmen. Nach dem Legen einer verschließbaren Flexüle in die V. jugularis und der Gewinnung einer Ausgangsprobe zur Bestimmung der Ba- 
siswerte wurde $1 \mathrm{~g}$ Glukose / $\mathrm{kg}^{0,75}$ metabolische Körpermasse innerhalb von 1 bis 3 Minuten in Form einer 40 \%igen körperwarmen Glukoselösung injiziert. Nach der Glukoseinfusion wurden im Abstand von 7 Minuten 9 weitere Blutproben zur Erfassung der Insulin- und Glukosereaktion gewonnen. Zur Plasmagewinnung wurden die Blutproben innerhalb von zwei Stunden zentrifugiert. Das Plasma wurde bis zur Laboruntersuchung bei minus $18^{0} \mathrm{C}$ gelagert.

Die Plasmaglukose wurde mittels GOD-POD-Methode der Fa. Boeringer-MannheimGmbH am automatischen Photometer CORONA der Fa. Clinicon bestimmt. Die Glukosehalbwertheit wurde mit geringer Abweichung von der Empfehlung von KANEKO (1997) (15. bis 45. min) mittels Regression aus den In-transformierten Glukosekonzentrationen von der 14. bis zur 42. min. bestimmt. Die Glukosehalbwertzeit ist mit Hilfe der fraktionierten Turnoverrate $\mathrm{k}=(\ln \mathrm{G} 1-\ln \mathrm{G} 2) /(\mathrm{T} 2-\mathrm{T} 1)^{*} 100=\% / \mathrm{min}$. berechnet worden, wobei lnG1 und lnG2 die ln-transformierten Glukosekonzentrationen in der 14. und 42. min. p. inj. und der Ausdruck (T 2 - T 1) die Zeitspanne zwischen der 42. und 14. min. sind. Aus k ließ sich die Glukosehalbwertzeit durch die Beziehung $\mathrm{G}_{\mathrm{HWZ}}=\ln 2 / \mathrm{k}^{*} 100$ in min errechnen. Als Ersatzparameter für die Flächen unter den Glukosekonzentrationsverlaufskurve nach dem Glukosestimulus wird das Flächenäquivalent $G_{A}$ verwendet. Die Bildung der Flächenäquivalente erfolgt durch Addition der Glukosekonzentration in der 2. bis 10. Probe abzüglich der Glukoseausgangskonzentration. Die Flächenäquivalente werden ohne Einheiten angegeben. Die maximale Glukosekonzentration über dem Basalspiegel $G_{M A X}$ ist die Glukosekonzentration in der 1. Probe $\left(G_{\max 1}\right)$ nach der Glukoseinfusion (7. min p. inj.) abzüglich der Glukoseausgangskonzentration $\left(\mathrm{G}_{0}\right)$ und wird in mmol/l angegeben. Weitere methodische Ausführungen liegen bei BURKERT (1998), STAUFENBIEL et al. (1999) und PANICKE et al. (2001) vor. Im Vergleich der metabolischen Parameter des GlukoseToleranz-Testes (GTT) der Jungbullen und Kühe wird die Signifikanz der Differenzen mit den p-Werten des t-Testes geprüft.

\section{Ergebnisse}

Bei den 292 untersuchten Jungbullen mit einer Körpermasse um 450 kg zum Untersuchungszeitpunkt konnten stabile Ergebnisse für alle metabolischen Merkmale des Glukose-Toleranz-Testes (GTT) innerhalb der Geburtsjahrgänge erreicht werden. Die Mittelwerte lagen für die Glukosebasalwerte der Ausgangskonzentration im Blut um $\mathrm{G}_{0}=4,5 \mathrm{mmol} / \mathrm{l}$, die Glukosemaximalwerte nach Infusion um $\mathrm{G}_{\mathrm{MAX}}=13,7 \mathrm{mmol} / \mathrm{l}$, die Glukoseflächenäquivalente $u m \mathrm{G}_{\mathrm{A}}=37,2$ sowie die Glukosehalbwertzeiten um $\mathrm{G}_{\mathrm{HWZ}}=47,8 \mathrm{~min}$. (Tab. 1).

Table 1

Ergebnisse des Glukose-Toleranz-Testes ( GTT ) bei Jungbullen im dritten Lebenshalbjahr (Results of GTT in young bulls according to test in the third life half year)

\begin{tabular}{|c|c|c|c|c|c|c|c|c|c|c|c|}
\hline \multirow[t]{2}{*}{ Jahr } & \multirow[b]{2}{*}{$\mathrm{n}$} & \multicolumn{2}{|c|}{ Gewicht kg } & \multicolumn{2}{|c|}{$\mathrm{G}_{0} \mathrm{mmol} / \mathrm{l}^{\$}$} & \multicolumn{2}{|c|}{$\mathrm{G}_{\underline{\text { MAX }}} \mathrm{mmol} / \mathrm{l}^{++}$} & \multicolumn{2}{|c|}{$\mathrm{G}_{\mathrm{A}}{ }^{+}$} & \multicolumn{2}{|c|}{$\mathrm{G}_{\mathrm{HWZ}} \min \#$} \\
\hline & & $x$ & $\mathrm{~s}$ & $X$ & $\mathrm{~s}$ & $x$ & $\mathrm{~S}$ & $x$ & $\mathrm{~s}$ & $X$ & $\mathrm{~S}$ \\
\hline 1999 & 96 & 460 & 48,4 & 4,4 & 0,6 & 13,4 & 1,4 & 37,2 & 6,7 & 49,9 & 14,4 \\
\hline 2000 & 105 & 447 & 45,8 & 4,4 & 0,7 & 13,7 & 1,8 & 37,4 & 7,6 & 47,6 & 15,7 \\
\hline 2001 & 91 & 443 & 36,8 & 4,6 & 0,5 & 14,1 & 1,5 & 36,7 & 7,2 & 45,7 & 10,5 \\
\hline alle & 292 & 450 & 44,5 & 4,5 & 0,6 & 13,7 & 1,6 & 37,1 & 7,2 & 47,8 & 13,9 \\
\hline
\end{tabular}


Table 2

Ergebnisse des Glukose-Toleranz-Testes (GTT) bei Kühen in der Laktation gemessen von der zweiten Woche a.p. bis zur 36. Woche p.p. (GTT in dairy cows in lactation in the $2^{\text {th }}$ week ante partum to $36^{\text {th }}$ week post partum)

\begin{tabular}{|c|c|c|c|c|c|c|c|c|c|c|c|}
\hline Laktations- & $\mathrm{n}$ & Gewi & $\mathrm{kg}$ & $\underline{\mathrm{G}}_{0}$ & $101 / 1^{\$}$ & $\underline{G}_{\text {MAX }}$ & $\mathrm{mol} / \mathrm{l}^{++}$ & $\underline{G}_{A^{+}}$ & & $\underline{G}_{\mathrm{HWZ}}$ & in\# \\
\hline woche & & $X$ & $\mathrm{~S}$ & $x$ & $\mathrm{~S}$ & $x$ & $\mathrm{~S}$ & $x$ & $\mathrm{~S}$ & $x$ & $\mathrm{~S}$ \\
\hline 2 w. a.p. & 10 & 581 & 62,6 & 3,7 & 0,3 & 11,2 & 1,0 & 51,9 & 2,3 & 60,3 & 12,7 \\
\hline partus & 28 & 552 & 38,7 & 4,0 & 0,4 & 11,1 & 1,4 & 47,2 & 6,9 & 43,1 & 8,5 \\
\hline 4 w.p.p. & 10 & 542 & 34,6 & 3,4 & 0,4 & 11,2 & 1,6 & 45,2 & 5,7 & 37,7 & 5,6 \\
\hline 8 w. p.p. & 10 & 566 & 23,2 & 4,0 & 0,5 & 11,7 & 1,9 & 49,3 & 7,9 & 48,9 & 18,0 \\
\hline 15 w. p.p. & 10 & 542 & 31,9 & 3,9 & 0,3 & 12,0 & 1,8 & 53,0 & 9,3 & 55,5 & 24,4 \\
\hline 26 w. p.p. & 8 & 548 & 28,2 & 3,7 & 0,4 & 11,6 & 0,5 & 50,2 & 3,4 & 51,0 & 11,0 \\
\hline 36 w. p.p. & 10 & 560 & 41,1 & 4,2 & 0,4 & 12,3 & 0,9 & 55,4 & 7,3 & 75,0 & 38,6 \\
\hline alle & 86 & 556 & 37,2 & 3,9 & 0,4 & 11,6 & 1,3 & 50,3 & 6,1 & 53,1 & 17,0 \\
\hline
\end{tabular}

Bei den Kühen in der Laktation blieben die Glukosekonzentration und die erreichte Maximalkonzentration nach Infusion um $\mathrm{G}_{0}=3,9 \mathrm{mmol} / \mathrm{l}$ und $\mathrm{G}_{\mathrm{MAX}}=11,6 \mathrm{mmol} / \mathrm{l}$ stabil und geringer als bei den Bullen. Die Körpermasse, das Glukoseflächenäquivalent und die Glukosehalbwertzeit verändern sich im Laktationsverlauf entsprechend der Leistungserwartung respective um die $556 \mathrm{~kg}$ Körpermasse sowie um $\mathrm{G}_{\mathrm{A}}=50$ und um $\mathrm{G}_{\mathrm{HWZ}}=53$ min. (Tab. 2).

\section{Diskussion}

Für eine zusätzliche Empfehlung zum Pedigreezuchtwert (PBV) zur Bewertung von Zuchtbullen vor der Nachkommenprüfung wird der Glukosehalbwertzeit $\mathrm{G}_{\mathrm{HWZ}}$ aus dem Glukose-Toleranz-Testes (GTT) wesentliche Bedeutung beigemessen (PANICKE et al., 2000a, 2001, 2002; FISCHER et al., 2003). Bei einer kombinierten Berücksichtigung beider Informationsquellen, der GTT-Parameter und des Pedigreezuchtwertes (PBV) im kalkulierten Zuchtwert (CBV) ist ein Informationszuwachs vor dem Testeinsatz (Tab. 3) zur Selektion unterlegener Bullen zu erwarten (PANICKE et al., 2002a; FISCHER et al., 2003). Die Information aus der Schätzung des kalkulierten Erwartungswertes (CBV) ist vier Jahre vor dem Nachkommenzuchtwert verfügbar.

Tabelle 3

Korrelationen zwischen Pedigree-Zuchtwert (PBV), geschätzten Erwartungswert (CBV) und dem Nachkommenzuchtwert (EBV) (Lebenshalbjahr 3; $n=28$ ) (Correlations between the pedigree breeding values (PBV), the predicted calculated breeding-values (CBV) and the offspring's breeding values (EBV); life half year 3; n=28)

\begin{tabular}{ccccccc}
\hline Merkmal & \multicolumn{5}{c}{ Nachkommenzuchtwert (EBV; August 2002) } \\
& Milch-kg & Fett-kg & Eiweiß-kg & Fett-\% & Eiweiß-\% & RZM \\
\hline PBV & $0,76^{* *}$ & 0,28 & $0,48^{*}$ & $0,72 * *$ & $0,61 * *$ & 0,12 \\
CBV & $0,84^{* *}$ & $0,57 * *$ & $0,68^{* *}$ & $0,76 * *$ & $0,65 * *$ & $0,53 * *$ \\
\hline${ }^{*}=\mathrm{p}<0,05 ; * *=p<0,01$ statistisch gesichert & \multicolumn{7}{c}{ ** }
\end{tabular}

Die Glukosebasiskonzentration $\mathrm{G}_{0}$ mit einem geringen Variationskoeffizienten um etwa $10 \%$ ist auf grund der Homöostase relativ stabil. Das Glukoseflächenäquivalent $\mathrm{G}_{\mathrm{A}}$ nach Glukoseapplikation drückt den Regelaufwand zur Wiederherstellung der Glukosehomöostase aus. Die Glukosehalbwertzeit $\mathrm{G}_{\mathrm{HwZ}}$ ist ein Ausdruck des Stoffwechselreaktionsvermögens der Kühe. Der altersphysiogische und metabolische Status der Jungrinder beeinträchtigt die Messung der metabolischen Parameter des Glukosetoleranztestes sowohl nach eigenen Ergebnissen (PANICKE et al., 2003; MÜLLER et al., 2003; PANICKE et al., 2001; STAUFENBIEL et al., 1999) als auch anderen Autoren 
wie MELENDEZ et al. (2002), ORDWAY et al. (2002), VOIGT et al. (1973), HAYIRLI et al. (2002) und GAAFAR et al. (2002). Der Alterseinfluss wurde an Zuchtbullen im Alter von 6 Monaten bis zu 6 Jahren sowohl auf die metabolischen Parameter des Glukose-Toleranz-Testes (GTT) (BURKERT, 1998) als auch auf ihre Beziehungen zum Nachkommenzuchtwert quantifiziert. Davon wird die Vergleichbarkeit der Tiere und damit die Eignung des Parameters für die Vorselektion betroffen. Im Vergleich zu anderen Altersabschnitten werden im dritten Lebenshalbjahr die besten Beziehungen mit mittleren Korrelationen um $r=0,4-0,5$ zu den Ertragsmerkmalen im Nachkommenzuchtwert wie für die Eiweißmenge oder den Relativzuchtwert Milch (RZM) erreicht, (PANICKE et al., 2001) (Tab. 4). Das favorisiert die Eignung dieses Altersabschnittes als Prüfzeitraum. Unterstützung findet dieser Prüfzeitraum auch durch umfangreiche Untersuchungen zur somtotropen Achse für das Wachstum und die Reproduktion von SAUERWEIN (1994), die das Wachstumshormon (GH) und die insulinartigen Wachstumsfaktoren (IGFs) einschließen. Rinder erreichen danach ein Plateau der Plasmawerte im Alter von zwei Jahren. Dies bleibt bei männlichen Tieren auch im Alter erhalten, während es bei weiblichen Tieren den Schwankungen durch Gravidiät und Laktation unterliegt (SAUERWEIN, 1994). Auch die genetische Struktur kann zur Priorität eines altersphysiologischen Prüfzeitpunktes durch imprintierte Loci beitragen. GEORGES (2002) berichtete dazu über das callipyge Gen (CLPG) beim Schaf. Es führt zur Muskelhypertrophy nach sechs Monaten, ist aber nach der Geburt nicht über die Genomanalyse nachzuweisen. Die molekulargenetischen Mechanismen werden in der Methylierung gesucht. COCKETT et al. (1996) wiesen die vererbte Mutation als Überdominanz auf dem Chromosom 18 nach. Insbesondere für die Wachstumsmerkmale wiesen DE VRIES et al. (1994) beim Schwein und ENGELLANDT (2002) für das Rind Imprintingeffekte nach, während für die Milchleistung KAISER (1998) keine solche Effekte nachweisen konnte.

Tabelle 4

Korrelationen zwischen der Glukosehalbwertzeit $\mathrm{G}_{\mathrm{HWZ}}$ der Bullen in den Lebenshalbjahren und ihren Zuchtwerten bei Schwarzbunten Bullen (ZWS Februar 2001, PANICKE et al., 2001a) (Correlations between of the parameter intravenous glucose test and estimated breeding value)

\begin{tabular}{llllllll}
\hline $\begin{array}{l}\text { Lebens- } \\
\text { halbjahr }\end{array}$ & Anzahl & \multicolumn{2}{l}{ Merkmale des Zuchtwertes ( EBV ) } \\
\hline 1 & 7 & Milch-kg & Fett-kg & Eiweiß-kg & Fett-\% & Eiweiß-\% & RZM \\
2 & 42 & $-0,62$ & $-0,30$ & $-0,50$ & $+0,28$ & $+0,24$ & $-0,44$ \\
$\mathbf{3}$ & $\mathbf{2 8}$ & $-\mathbf{0 , 5 2} * *$ & $+0,01$ & $-0,08$ & $-0,01$ & $-0,12$ & $-0,06$ \\
4 & 25 & $+0,36$ & $+0,43^{*}$ & $-\mathbf{0 , 4 0 *}$ & $+0,26$ & $+\mathbf{0 , 4 6}$ & $-\mathbf{0 , 4 4}$ \\
5 & 37 & $-0,22$ & $-0,32$ & $+0,29$ & $-0,13$ & $-0,22$ & $+0,29$ \\
6 & 45 & $-0,12$ & $-0,08$ & $+0,01$ & $+0,08$ & $+0,28$ & $-0,01$ \\
7 & 35 & $+0,03$ & $+0,19$ & $+0,09$ & $+0,16$ & $+0,11$ & $+0,12$ \\
8 & 28 & $+0,18$ & $+0,17$ & $+0,20$ & $-0,04$ & $-0,04$ & $+0,20$ \\
9 & 24 & $+0,02$ & $-0,27$ & $+0,05$ & $-0,28$ & $+0,02$ & $-0,04$ \\
$\geq 10$ & 21 & $+0,15$ & $+0,41$ & $+0,19$ & $+0,16$ & $+0,01$ & $+0,24$ \\
\hline alle & 292 & $-0,07$ & $-0,06$ & $-0,02$ & $+0,02$ & $+0,11$ & $-0,04$ \\
\hline$*=\mathrm{p}<0,05 ; * *=\mathrm{p}<0,01$ statistisch gesichert & \multicolumn{7}{c}{}
\end{tabular}

Nach eigenen Untersuchungen sinkt die Glukosehalbwertzeit $\left(\mathrm{G}_{\mathrm{HwZ}}\right)$ mit dem Alter und der Körpermasse in dem untersuchten Lebensabschnitt bis zu 17 Monaten im Zwillingsversuch (MÜLLER et al., 2003; PANICKE et al., 2003) (Tab. 5). Die größte fütterungsbedingte Differenz wird im Alter von 12 Monaten ausgewiesen. Exakt zu diesem Zeitpunkt konnten nach eigenen Ergebnissen (PANICKE et al., 2001) die bes- 
ten Beziehungen um $\mathrm{r}=0,4-0,5$ zur Nachkommenleistung der Töchter der untersuchten Bullen geschätzt werden. Mit den Ergebnissen des Zwillingsversuches wird der Einfluss des Alters und der Ernährungsintensität auf die metabolischen Merkmale des Glukose-Toleranz-Testes (GTT) quantitativ gemessen. Wichtige Voraussetzungen für die Messung physiologischer Merkmale sind eine bedarfsgerechte Fütterung und der optimale Messzeitpunkt bei einem Alter um 12 Monate für die $\mathrm{G}_{\mathrm{HwZ}}$., um die Vergleichbarkeit zwischen den Individuen zu gewährleisten.

Tabelle 5

Ergebnisse des Glukose-Toleranz-Testes ( GTT ) an Zwillingspaaren bei differenzierter Fütterung (PANICKE et al., 2003) (Results of the GTT of twin pairs by varied feeding)

\begin{tabular}{|c|c|c|c|c|c|c|c|c|}
\hline Altersgruppe & $\begin{array}{l}\text { Gewicht } \\
\text { intensiv }\end{array}$ & $\begin{array}{l}\text { g } \\
\text { extensiv }\end{array}$ & $\begin{array}{l}\mathrm{G}_{\mathrm{HWZ}}{ }^{*} \\
\text { intensiv }\end{array}$ & $\begin{array}{l}\min \\
\text { extensiv }\end{array}$ & $\begin{array}{l}\mathrm{G}_{\mathrm{MAX} 1}{ }^{++} \\
\text {intensiv }\end{array}$ & $\begin{array}{l}\mathrm{mmol} / \mathrm{l} \\
\text { extensiv }\end{array}$ & $\begin{array}{l}\mathrm{G}_{\mathrm{A}} * * * \\
\text { intensiv }\end{array}$ & extensiv \\
\hline \multicolumn{9}{|l|}{6 Wochen $n=9$} \\
\hline Mittelwert & 49,2 & 48,8 & 37,6 & 40,8 & 12,9 & 12,8 & 33,2 & 37,4 \\
\hline $\begin{array}{l}\text { Stand.abw. } \\
6 \text { Monate } n=15\end{array}$ & 5,5 & 6,4 & 14,7 & 13,1 & 1,4 & 0,9 & 7,2 & 7,9 \\
\hline Mittelwert & 149,9 & 141,1 & 40,9 & 40,0 & 13,4 & 13,9 & 36,7 & 43,7 \\
\hline $\begin{array}{l}\text { Stand.abw. } \\
12 \text { Monate n=15 }\end{array}$ & 21,9 & 22,5 & 9,0 & 9,7 & 1,1 & 1,1 & 7,6 & 9,0 \\
\hline Mittelwert & 315,7 & 255,7 & 26,6 & 34,4 & 13,2 & 13,2 & 25,1 & 30,1 \\
\hline $\begin{array}{l}\text { Stand.abw. } \\
17 \text { Monate } n=7\end{array}$ & 33,8 & 39,9 & 10,6 & 6,5 & 1,5 & 1,1 & 9,1 & 6,1 \\
\hline Mittelwert & 446,0 & 346,9 & 25,7 & 26,4 & 14,3 & 13,0 & 22,8 & 15,0 \\
\hline Stand.abw. & 26,3 & 13,5 & 5,2 & 9,7 & 1,1 & 0,6 & 7,9 & 10,2 \\
\hline
\end{tabular}

;\# = $\mathrm{G}_{\mathrm{HWZ}}=$ Glukose-Halbwertzeit, ++= $\mathrm{G}_{\mathrm{MAX}}=$ Glukosemaximalwert nach Infusion, $+=\mathrm{G}_{\mathrm{A}}=$ Glukosefläche

Im Vergleich (Tab. 6) unterscheiden sich die metabolischen Merkmale des GTT der Jungbullen im Mittel der Jahre und der laktierenden Kühe im Mittel der Laktationsabschnitte für Körpermasse, Glukosebasiskonzentration $\mathrm{G}_{0}$, der Maximalkonzentration nach Infusion $\mathrm{G}_{\mathrm{MAX}}$ und der Glukosefläche $\mathrm{G}_{\mathrm{A}}$ hoch signifikant voneinander. Für die Glukose-Halbwertzeit $\mathrm{G}_{\mathrm{HWZ}}$ fällt die Signifikanz geringer aus. Ihr Variationskoeffizient um $30 \%$ ist wesentlich höher als bei den übrigen Merkmalen. Das zu den Jungbullen untersuchte Vergleichsniveau der laktierenden Kühe in der Glukosebasiskonzentration in der vierten Laktationswoche von $\mathrm{G}_{0}=3,4 \mathrm{mmol} / \mathrm{l}$ wird auch mit 3,3 $\mathrm{mmol} / \mathrm{l}$ bei ORDWAY et al. (2002) und GAAFER et al. (2002) bei einem hohen Leistungsniveau von 12489 kg Milch, 433 Fett-kg und 398 Protein-kg in der Laktation (ORDWAY et al., 2002) bzw. $41.8 \mathrm{~kg}$ Milch/Tag bei GAAFER et al. (2002) ausgewiesen. GAAFER et al. (2002) belegen auch in der 6. und 14. Laktationswoche ein vergleichbares Glukose-Niveau von $\mathrm{G}_{0}=3,7 \mathrm{mmol} / \mathrm{l}$.

Die Glukose-Halbwertzeit $G_{H W Z}$ der Jungbullen im dritten Lebenshalbjahr von 47,8 min unterscheidet sich nicht von der Glukose-Halbwertzeit $G_{\text {HwZ }}$ der Kühe in der 8. Laktationswoche von 48,9 min. Zu diesem Zeitpunkt ist bei den Kühen aufgrund der höchsten Milchleistung ein hoher Glukosebedarf zu erwarten, dem Kühe mit hoher Leistung gerecht werden. Exakt zu diesem Zeitpunkt in der 8. Laktationswoche der Kühe weist CASSEL (2001) nach VEERKAMP und KOENEN (1999) die engste genetische Korrelation zwischen Energie-Balance und Ertrag mit $r_{g}=-0,84$ bei fast unabhängiger Variation zur Trockenmasseaufnahme mit $r_{g}=0,06$ und einem mittleren Heritabilitätskoeffizienten von $\mathrm{h}^{2}=0,20$ aus (Tab. 7). 
Tabelle 6

Differenzen der Ergebnisse des Glukose-Toleranz-Testes ( GTT ) bei Jungbullen im dritten Lebenshalbjahr und bei Kühen in der Laktation gemessen von der zweiten Woche a.p. bis zur 36. Woche p.p. (Difference of results GTT in young bulls according to test in the third life half year and the dairy cows in lactation according to in the $2^{\text {th }}$ weeks ante partum to $36^{\text {th }}$ weeks post partum)

\begin{tabular}{|c|c|c|c|c|c|c|c|c|c|c|c|}
\hline & \multirow[b]{2}{*}{$\mathrm{n}$} & \multicolumn{2}{|c|}{ Gewicht kg } & \multicolumn{2}{|c|}{$\underline{\mathrm{G}}_{0} \mathrm{mmol} / \mathrm{l}^{\$}$} & \multicolumn{2}{|c|}{$\underline{\mathrm{G}}_{\mathrm{MAX}} \mathrm{mmol} / \mathrm{l}^{++}$} & & \multicolumn{2}{|c|}{$\underline{G}_{\mathrm{HWZ}} \min \#$} \\
\hline & & $x$ & $\mathrm{~s}$ & $x$ & $\mathrm{~s}$ & $X$ & $\mathrm{~s}$ & $X$ & $\mathrm{~S}$ & $x$ & $\mathrm{~S}$ \\
\hline Kühe 4. LW & 10 & 542 & 34,6 & 3,4 & 0,4 & 11,2 & 1,6 & 45,2 & 5,7 & 37,7 & 5,6 \\
\hline Kühe 8. LW & 10 & 566 & 23,2 & 4,0 & 0,5 & 11,7 & 1,9 & 49,3 & 7,9 & 48,9 & 18,0 \\
\hline Lakt-Mittel & 86 & 556 & 37,2 & 3,9 & 0,4 & 11,6 & 1,3 & 50,3 & 6,1 & 53,1 & 17,0 \\
\hline Jungbullen & 292 & 450 & 44,5 & 4,5 & 0,6 & 13,7 & 1,6 & 37,2 & 7,2 & 47,8 & 13,9 \\
\hline Differenz & & -104 & & $+0,6$ & & $+2,1$ & & $-13,1$ & & $-5,8$ & \\
\hline $\mathrm{p}^{-W e r t}{ }^{\S}$ & & 0,001 & & 0,001 & & 0,001 & & 0,001 & & 0,01 & \\
\hline
\end{tabular}

$\mathrm{LW}=$ Laktationswoche, ${ }^{s}=\mathrm{G}_{0}=$ Glukosebasiskonzentration; ++= $\mathrm{G}_{\mathrm{MAX}}=$ Glukosemaximalwert nach Infusion ,

$+=\mathrm{G}_{\mathrm{A}}=$ Glukosefläche, \# = $\mathrm{G}_{\mathrm{Hwz}}=$ Glukose-Halbwertzeit, ${ }^{\S}=$ Signifikanz $\mathrm{p}<\ldots$,

Tabelle 7

Genetische Parameter der Energie -Balance bei Milchkühen (Aufnahme-Leistungs+Erhaltungsbedarf), (CASSEL, 2001, nach VEERKAMP und KOENEN, 1999) (Genetic parameters of trait related to energy-balance in dairy cows, CASSEL, 2001, adapted VEERKAMP und KOENEN, 1999)

\begin{tabular}{lccc}
\hline & Energie-Balance bei Kühen & \multicolumn{2}{c}{$\begin{array}{l}\text { Genetische Korrelation mit } \\
\text { Trockenmasse } \\
\text { aufnahme }\end{array}$} \\
$\begin{array}{l}\text { Merkmal im } \\
\text { Laktationszeitpunkt }\end{array}$ & $\mathrm{h}^{2}$ & Ertrag & 0,01 \\
\hline 2.-6.Lakt.-Woche & 0,15 & $-0,64$ & $\mathbf{0 , 0 6}$ \\
\hline 7.-12.Lakt.-Woche & $\mathbf{0 , 2 0}$ & $-\mathbf{0 , 8 4}$ & 0,14 \\
\hline 13.-18 Lakt.-Woche & 0,21 & $-0,80$ & 0.43 \\
\hline 19.-24.Lakt.-Woche & 0,19 & $-0,40$ & 0.55 \\
\hline 1.-100. Lakt.-Tag & 0,51 & $-0,46$ & - \\
\hline Mittel der 26 Wochen & 0,35 & $-0,37$ & \\
\hline
\end{tabular}

Das Prüfniveau für die Methode des Glukose-Toleranz-Testes (GTT) wird einerseits dem Leistungsbedarf gerecht. Einer größeren Infusionsmenge steht andererseits als Limit die Nierenschwelle gegenüber. Nicht abgebaute applizierte Glukose wird bei Rindern nach KANEKO (1997) bei einer Blutkonzentration von mehr als $10 \mathrm{mmol} / \mathrm{l}$ oder 180mg/dl über die Nieren ausgeschieden. Das untersuchte Prüfniveau des Glukose-Toleranz-Testes (GTT) wird beiden Anforderungen dem Bedarf und der Nierenschwelle gerecht. Daraus kann geschlussfolgert werden, dass durch den Glukose-Toleranz-Testes (GTT) mit dem Abbau der Glukoseinfusion bei den Jungrindern im Umkehrschluss die genetische Ausstattung zur Steuerung der Glukosebereitstellung bei erhöhtem Bedarf in der Laktation gemessen werden kann. Mit der Infusionsmenge zum Testzeitpunkt der Jungbullen wird der Bedarf imitiert, um die genetische Ausstattung für die Steuerung des Stoffwechselreaktionsvermögen seiner Töchter unter der erwarteten Leistung zu prüfen. Damit finden das Glukoseprüfniveau und die Glukosehalbwertzeit $\mathrm{G}_{\mathrm{HWZ}}$ als geeigneter Parameter bei Jungbullen Bestätigung.

Wenn sich die bisher erzielten Ergebnisse bestätigen, könnte eine zusätzliche Empfehlung für die Bewertung der Zuchtbullen vor dem Testeinsatz gegeben werden. Es wären weniger Bullen in die Nachkommenprüfung einzustellen und damit Prüfkosten zu sparen oder bei gleichen Kosten könnte die Selektionsintensität erhöht werden. In kleinen Populationen kann damit die Effizienz eines Jungtierprogramms wesentlich verbessert werden. 


\section{Danksagung}

Die Autoren danken dem Ministerium für Bildung, Wissenschaft und Kultur des Landes Mecklenburg-Vorpommern für die finanzielle Unterstützung dieses Forschungsprojektes.

\section{Literatur}

BURKERT, O.:

Untersuchungen zum intravenösen und modifizierten Glukosetoleranztest bei Zuchtbullen. Freie Universität Berlin, Diss., 1998

CASSELL, B.G.:

Optimal Genetic Improvement for the High Producing Cow. J. Dairy Sci. 84 (2001) E. Suppl., 144-150

COCKETT, N.E.; JACKSON, S.P.; SHAY, T.L et al.: Polar Overdominance at the Ovine callipyge Locus. Science 273 (1996), 236-238

DeVRIES, A.G.; KERR, A.; TIER, B.; LONG, T.; MEUWISSEN, T.H.E.:

Genetic imprinting effects on rate and compos. of pig growth. Theor. Appl. Genet. 88 (1994), 10371042

ENGELLANDT, T.; TIER, B.:

Genetic variances due to imprinting genes in cattle. J. Anim. Breed. Genet. 119 (2002), 154-165

EULITZ-MEDER, C.; FUHRMANN, H.; SALLMANN, H.-P.; GELDERMANN, H.:

Stoffwechselbelastungstests beim Milchrind zur Beurteilung der Leistungsveranlagung. Deutsche Tierärztliche Wochenschrift 95 (1988) 7, 268-271

FISCHER, E.; STAUFENBIEL, R.; PANICKE, L.:

Metabolische Merkmale des Glukosetoleranztestes (GTT) zur zusätzlichen Bewertung von Jungbullen. Arch. Tierz., Dummerstorf 46 (2003a) im Druck

GEORGES, M.; BARALDI, F.; BUYS, N.; CHARLIER, C.; COLETTE, C.; DAVIS, E.; DI SILVESTRO, F.;

MOREAU, L.; NEZER, C.; NGUYEN, M.; SEGERS, K.; SHAY, T.; SMIT, M.; COCKETT, N.: On the Contribution of Imprinted Loci to Variation in Animal Body Composition. Communication 92 (2002), 111

GAAFAR, K.M.; HAGEMEISTER, H.; SCHÖNHUSEN, U.; KANITZ, W.; SOUFFRANT, W.B.; GABEL, M.; VOIGT, J.:

The effect of substitution of starch by protected fat on the level of plasma free amino acids of dairy cows. Proc. Soc. Nutr. Physiol. (1992) 11, 136

HAYIRLI, A.; BERTICS, S.J.; GRUMMER, R.R.:

Effects of Slow-release Insulin on Production, Liver Triglyceride, and Metabolic Profiles of Holsteins in Early Lactation. J. Dairy Sci. 85 (2002), 2180-2191

KAISER, C.J.; GODDARD, M.E.; REVERTER, A.:

Analysis of genetic imprinting effects for test day milk yield in Australian Holstein Cattle. Proc. $6^{\text {th }}$ World Congress on Genetics applied to Livestock Production, January 11-16, Armidale, Australia, Vol. 23 (1998), 355-358

KANEKO, J.J.; HARVEY, J. W.; BRUSS, M.L.:

Carbohydrate metabolism and its diseases Clinical Biochemistry of Domestic Animals. Academic Press, $5^{\text {th }}$ Ed. 1997, 65

KIRCHGESSNER, M.:

Tierernährung, Leitfaden, Beratung und Praxis. Verlags Union Agrar, Frankfurt/M., 1997, 55

LØVENDAL, P.; NIELSEN, M.; VAN DER WERF, J.H.J.:

Genetic parameters for glucose induced insulin release in juvenile dairy cattle. $46^{\text {th }}$ Annual Meeting of the EAAP, G 2.10, p. 19, Prag, 1995

MELENDEZ, P.; DONOVAN, A.; RISCO, C.A.; HALL, M.B.; LITTELL, R.; GOFF, J.:

Metabolic Responses of Transition Holstein Cows Fed Anionic Salts and Supplemented at Calving with Calcium and Energy. J. Dairy Sci. 85 (2002), 1085-1092

MIN, S.H.; McCUTCHEON, S.N.; MACKENZIE, D.D.S.; WICKHAM, B.W.:

Plasma metabolite and hormone concentation in Friesian calves of low or high genetic merit: effects of sex and age. Anim. Prod. 56 (1993), 17-27

MÜLLER, U.; HOESSLER, J.; HASSELMANN, L.; BETZIN, S.; PANICKE, P.; STAUFENBIEL, R.: Influencing Factors on Growth and Back Fat Thickness in Dairy Heifers - A Twin Study. $12^{\text {th }}$ International conference Current Problems of Breeding, Health, Growth and Production of Cattle. 18-19 February 2003, Ceské Budejovice, Czech Republic, im Druck 
OLBRICH-BLUDAU, A.; SCHAMS, D.; SCHALLENBERGER, E.; GRAML, R.; PIRCHNER, F.:

Relationship between age, nutritional status and dairy merit with plasma profiles of growth hormone (GH) and free fatty acids (FFA) in bulls. J. Anim. Breed. Genet. 110 (1993) 3, 171-181

OLDENBROEK, J.K.; GALESLOOT, P.A.J.; POOL, M.H.; VAN DER WERF, J.H.J.:

Effects of selection for milk yield on feed intake and metabolism of heifers in early lactation. $48^{\text {th }}$. Annual Meeting of the EAAP, Wien, 1997

ORDWAY, R.S.; ISHLER, V.A.; VARGA, G.A.:

Effects of Sucrose Supplementation on Dry Matter Intake Milk Yield, and Blood Metabolites of Periparturient Holstein Cows. J. Dairy Sci. 85 (2002), 879-888

PANICKE, L.; STAUFENBIEL, R.; BURKERT, O.; FISCHER, E.; REINHARDT, F.:

Zusammenhang zwischen Parametern des Glukosetoleranztestes bei Jungbullen und deren Nachkommenzuchtwert (EBV). Arch. Tierz., Dummerstorf 43 (2000a) 3, 231-239

PANICKE, W.; STAUFENBIEL, R.; BURKERT, O.; FISCHER, E.; REINHARDT F.:

Relationship between parameters of the glucose tolerance test (GTT) in young sires and their estimated breeding value (EBV). 51 $1^{\text {st }}$ EAAP Meeting 21-24 August 2000b, The Hague, The Netherlands, G5.5, 85

PANICKE, L.; STAUFENBIEL, R.; BURKERT, O.; FISCHER, E.; REINHARDT, F.:

Relationships between parameters of the glucose tolereance test (GTT) in young sires and their estimated breeding values (EBV) J. Dairy Sci. 83 (2000c) 1, 49

PANICKE, L.; FISCHER, E.; STAUFENBIEL, R.:

Variation und Eignung von physiologischen Merkmalen des Glukosetoleranztestes für die indirekte Eigenleistungsprüfung von Jungbullen. Arch. Tierz., Dummerstorf 44 (2001) 4, 381-394

PANICKE, L.; STAUFENBIEL, R.; FISCHER, E.:

Additional evaluation of the parameters of the glucose tolerance test (GTT) in connection with pedigree performance (PBV) in growing young bulls. J. Dairy Sci. 85 (2002) 1, 34

PANICKE L.; MÜLLER, U.; BEHN, H.; STAUFENBIEL, R.; OPRZADEK, A.:

Metabolic Statuses at the Metabolic Traits in the Glucose Tolerance Test (GTT). $12^{\text {th }}$ International conference Current Problems of Breeding, Health, Growth and Production of Cattle. 18-19 ${ }^{\text {th }}$ February 2003, Ceské Budejovice, Czech Republic, im Druck

REINECKE, P.; SEELAND, G.:

Untersuchungen zur Eignung der Schilddrüsenaktivität als Frühselektionskriterium für Merkmale des Wachstums und der Milchleistung beim Rind. Humboldt-Universität Berlin, Diss. B, 1985

REINICKE, U.:

Der intravenöse und modifizierte Glukosetoleranztest beim Milchrind - Einflußfaktoren und Beziehungen zur Milchleistung. FU Berlin, Diss., 1993

ROBINSON, D.L.; HAMMOND, K.; MCDOWELL, G.H.:

Relationships between breeding values and physiological responses to fasting and refeeding in dairy bulls: update for young animals. J. Anim. Breed. Genet. 111 (1994), 257-264

SAUERWEIN, H.:

Die Bedeutung der somatropen Achse für Muskelwachstum und Reproduktion bei landwirtschaftlichen Nutztieren. Habilitationssschrift TU München Freising-Weihenstephan, Verlag Markus Hänsel HohenHausen, 1994

SEELAND, G.; REINECKE, G.; SCHÖNMUTH, G.; SEGAL, L.; LEUTHOLD, G.:

Beziehungen zwischen der Schilddrüsenaktivität weiblicher Jungrinder und ihrer späteren Milchleistung: 2. Mitt.: Schätzung genetischer und phänotypischer Parameter der Untersuchungsmerkmale. Arch. Tierz., Berlin 27 (1984), 235-245

SØRENSEN, M.K.; MADSEN, P.; SEJRSEN, K.; VESTERGAARD, M.; LOVENDAHL, P.:

Prediction of breeding value for milk, fat and protein yield based on endocrine response profiles. J. Dairy Sci. 83 (2000) 1, 201

STAUFENBIEL, R.:

Energie- und Fettstoffwechsel des Rindes unter besonderer Berücksichtigung der Messung der Rückenfettdicke und der Untersuchung von Fettgewebe. Habilitationsschrift Freie Universität Berlin, 1993

STAUFENBIEL, R.; REINICKE, U.; PANICKE, L.:

Zum Glukosetoleranztest beim Rind. 1.Mitt.: Beziehungen zum Laktationsabschnitt und zur Milchleistung. Arch. Tierz., Dummerstorf 42 (1999) 1, 45-56

VEERKAMP, R.F.; KOENEN, E.P.:

Genetics of Food intake, live weight condition score, and energy balance. Occas. Publ. No. 24, Br. Soc. Anim. Sci. (1999), 63-73

WEBER, J.: FU Berlin, 2003, unveröffentlicht

VOIGT, J.; PIATKOWSKI, B.; GIRSCHEWSKI, H.:

Untersuchung einiger Blutinhaltsstoffe gesunder und erkrankter Hochleistungskühe im geburtsnahen Zeitraum. Arch. Tierz., Berlin 16 (1973), 271-283 
XING, G.Q.; MACKENZIE, D.D.S.; McCUTCHEON, S.N.; WICKHAM, B.W.:

Pancreatic insulin responses to exogenous glucose in Friesian heifers of low or high genetic merit for milk-fat-yield. Anim. Prod. 56 (1993), 171-178

Eingegangen: 23.01.2003

Akzeptiert: 18.02.2003

Anschriften der Verfasser

Prof. Dr. LOTHAR PANICKE

Forschungsinstitut für die Biologie

Dr. ECKHARD FISCHER

landwirtschaftlicher Nutztiere (FBN)

Universität Rostock

Wilhelm-Stahl-Allee 2

Fachbereich Agrarökologie

D-18196 Dummerstorf

Justus-von-Liebig-Weg 6

D-18059 Rostock

Dr. BERND FISCHER

Institut für Landwirtschaft und Gartenbau

des Landes Sachsen - Anhalt

D-39606 Iden / Altmark

Prof. Dr. RUDOLF STAUFENBIEL

Freie Universität Berlin

Klinik für Klauentiere

Königsweg 65

D-14163 Berlin 